\title{
Ordemann, Jessica
}

\section{Soziale Herkunft, Statusreproduktion und Studium ohne Abitur}

formal und inhaltlich überarbeitete Version der Originalveröffentlichung in:

formally and content revised edition of the original source in:

Sozialer Fortschritt 67 (2018) 4, S. 269-282

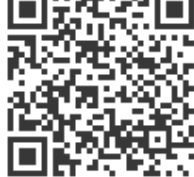

Bitte verwenden Sie in der Quellenangabe folgende URN oder DOI /

Please use the following URN or DOI for reference:

urn:nbn:de:0111-pedocs-161168

$10.25656 / 01: 16116$

https://nbn-resolving.org/urn:nbn:de:0111-pedocs-161168

https://doi.org/10.25656/01:16116

\section{Nutzungsbedingungen}

Gewährt wird ein nicht exklusives, nicht übertragbares, persönliches und beschränktes Recht auf Nutzung dieses Dokuments. Dieses Dokument ist ausschließlich für den persönlichen, nicht-kommerziellen Gebrauch bestimmt. Die Nutzung stellt keine Übertragung des Eigentumsrechts an diesem Dokument dar und gilt vorbehaltlich der folgenden Einschränkungen: Auf sämtlichen Kopien dieses Dokuments müssen alle Urheberrechtshinweise und sonstigen Hinweise auf gesetzlichen Schutz beibehalten werden. Sie dürfen dieses Dokument nicht in irgendeiner Weise abändern, noch dürfen Sie dieses Dokument für öffentliche oder kommerzielle Zwecke vervielfältigen, öffentlich ausstellen, aufführen, vertreiben oder anderweitig nutzen.

Mit der Verwendung dieses Dokuments erkennen Sie die Nutzungsbedingungen an.

\section{Terms of use}

We grant a non-exclusive, non-transferable, individual and limited right to using this document.

This document is solely intended for your personal, non-commercial use. Use of this document does not include any transfer of property rights and it is conditional to the following limitations: All of the copies of this documents must retain all copyright information and other information regarding legal protection. You are not allowed to alter this document in any way, to copy it for public or commercial purposes, to exhibit the document in public, to perform, distribute or otherwise use the document in public.

By using this particular document, you accept the above-stated conditions of use.

\section{Kontakt / Contact:}

\section{peDOCS}

DIPF | Leibniz-Institut für Bildungsforschung und Bildungsinformation Informationszentrum (IZ) Bildung

E-Mail: pedocs@dipf.de

Internet: www.pedocs.de

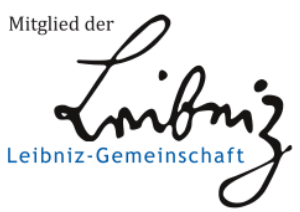




\title{
Soziale Herkunft, Statusreproduktion und Studium ohne Abitur
}

(Social Origins, Status Reproduction and Non-Traditionals)

\author{
Jessica Ordemann
}

\author{
Adresse \\ Deutsches Institut für Internationale Pädagogische Forschung (DIPF) \\ Warschauerstraße 34-38, 10243 Berlin \\ E-Mail: ordemann@dipf.de
}

\section{Zusammenfassung}

Der folgende Beitrag untersucht mit Daten der Erwachsenenkohorte SC6 7.0.0 des Nationalen Bildungspanels (NEPS), ob ein Studium ohne Abitur den Einfluss der sozialen Herkunft auf die späteren Statusplatzierungen in der Berufshierarchie abmindern kann. Die Analysen mit 146 Akademikern ohne Abitur und 628 Akademikern mit Abitur zeigen, dass die soziale Herkunft für Akademikern ohne Abitur aus statusniedrigen und statusmittleren Elternhäusern keine Rolle mehr spielt während sie für Akademiker ohne Abitur aus statushohen Elternhäusern noch eine, negative, Bedeutung hat.

\section{Keywords}

Studium ohne Abitur, Akademiker ohne Abitur, dritter Bildungsweg, beruflicher Status, Bildungserträge

\section{Abstract}

The following paper inquires if studying without an official university entrance certification decreases the relationship between social origin and status attainment for those non-traditionals. Using data of the starting cohort adults SC6 7.0.0 of the National Educational Panel Study (NEPS) I can show, that after five years in the labor market, the social origins of non-traditionals from the lower and middle classes have no effect on their status attainment, whereas it still effects - negatively - the status attainment of those non-traditionals from higher classes.

\section{Keywords}

Alumni, studies without university entrance certification, status attainment, yields on education, non-traditionals 


\section{Einleitung}

Zwischen sozialer Herkunft, gewähltem Bildungsweg und späteren Lebenschancen besteht seit langem ein enger Zusammenhang, der insbesondere für Akademiker von Bedeutung ist. Denn die soziale Herkunft wirkt an allen Übergängen auf dem Weg hin zum Abitur. Hochschulabsolventen mussten häufiger als andere Bildungsabsolventen die Wahl treffen, welchen Bildungsweg sie einschlagen und ob sie im allgemeinen Bildungssystem verbleiben oder nicht. An den Übergängen in die Sekundarstufe I und II und später in das tertiäre Bildungssystem wählen Kinder und Jugendliche aus statusniedrigeren Elternhäusern seltener den Weg, der sie zur formalen Hochschulzugangsberechtigung und damit zum Studium führt (vgl. u.a. Baumert/Schümer 2001; Ehmke/Baumert 2007; Maaz et al. 2008; Maaz et al. 2009; Maaz/Dumont 2018; Watermann et al. 2014). Der Zusammenhang zwischen Elternhaus und Bildungsweg der Kinder ist zwar in den letzten Jahren geringer geworden (vgl. u.a. Breen et al. 2009, 2010, 2012; Müller/Pollak 2010), jedoch haben sich die sozialen Disparitäten größtenteils auf den Übergang in die Hochschule verschoben und Kinder aus bildungsfernen Arbeiterfamilien nehmen nach wie vor seltener ein Studium auf (Lörz/Schindler 2011; Lörz 2013; Schindler/Lörz 2012). Da die Verzahnung zwischen Bildungssystem und Arbeitsmarkt in Deutschland besonders eng ist (Allmendinger 1989; Müller 2001; Müller/Gangl 2003), werden Bildungsabsolventen aus statusniedrigeren Elternhäusern systematisch aufgrund ihrer sozialen Herkunft von den besten Bildungserträgen und Lebenschancen der Akademiker ausgeschlossen.

Die Chancenungleichheiten im Bildungssystem, die zunehmende Relevanz von lebenslangem Lernen sowie die steigende gesellschaftliche Nachfrage nach Akademikern zur Deckung des Fachkräftemangels führten in den vergangenen Jahrzehnten zu einer schrittweisen Öffnung der Hochschulen für beruflich Qualifizierte ohne Abitur. Zuletzt öffnete die Kultusministerkonferenz (KMK) 2009 bundesweit den Hochschulzugang für alle Personen ohne Abitur, die entweder einen Meisterabschluss oder einen beruflich vergleichbaren Fortbildungsabschluss besaßen. Auch eine Berufsausbildung mit anschließender Berufserfahrung und Bestehen einer Eignungsprüfung oder eines Probestudiums ermöglichen den Zugang zu einer Hochschule (KMK 2009). Seither besteht eine formale Gleichberechtigung von beruflicher und akademischer Bildung in Bezug auf den Hochschulzugang, wenngleich 
nicht alle Bundesländer den KMK-Beschluss gleichwertig umgesetzt haben (Ulbricht 2012). Zudem nutzen die beruflich Qualifizierten ohne Abitur diesen Weg hin zu einem Studium nur in geringfügigem Maße (Nickel/ Duong 2012, Wolter et al. 2014) und brechen häufiger als andere Studierendengruppen das Studium ab (Dahm/Kerst 2016). Dennoch waren 2015 gut 1,2 Prozent aller beruflich vorqualifizierten Hochschulabsolventen ohne Abitur, d. h. 6.247 Personen, erfolgreich (Destatis 2016) und schlossen das Studium als Akademiker ohne Abitur ab. Für sie hat der Studienabschluss das Potential, soziale Disparitäten, die auf dem ersten Bildungsweg wirkten, zu verringern - wenn durch die politisch etablierte Chancengleichheit im Zugang zum Hochschulstudium auch eine Ergebnisgleichheit bei den Bildungserträgen der Akademiker ohne und mit Abitur nach dem Studienabschluss entsteht. Wäre dies nicht der Fall, dann würden sich die Bildungsungleichheiten von dem Bildungssystem in den Arbeitsmarkt verlagern.

Ob die Bildungserträge von Akademikern ohne Abitur denjenigen entsprechen, die das Studium mit Abitur aufgenommen haben, oder ob sich die bekannten sozialen Disparitäten der sozialen Herkunft von dem Zugang zum Hochschulabschluss in den Arbeitsmarkt verlagern, bleibt derzeit offen. Es gibt jedoch Hinweise darauf, dass Akademiker ohne Abitur, trotz gleichem Bildungszertifikat auf dem Arbeitsmarkt benachteiligt sein könnten. Grund dafür ist, dass die soziale Herkunft weit über das Bildungssystem in den Arbeitsmarkt hineinwirkt (Manzoni 2014) und selbst bei der Auswahl von promovierten Akademikern für Managementpositionen in Großunternehmen eine entscheidende Rolle spielt (Hartmann/ Kopp 2001). Da Studierende ohne Abitur vorwiegend aus statusniedrigeren Elternhäusern kommen (vgl. u.a. Brändle 2016; Diller et al. 2011; Rzepka 2016; Wolter et al. 2015) und im Studium geringere Leistungen zeigen als ihre Kommilitonen mit Abitur (Brändle/ Lengfeld 2015, 2017), liegt die Annahme nahe, dass sich die soziale Herkunft für sie trotz Hochschulabschluss weiterhin benachteiligend auf die Bildungserträge auswirkt. Hier setzt der vorliegende Artikel an und prüft, ob die soziale Herkunft der Akademiker auch nach einem Studium einen Einfluss auf ihre Bildungserträge hat und wie stark dieser verglichen mit den Akademikern mit Abitur ist. Hierfür wird der Vergleich der Statusplatzierungen der Akademiker ohne und mit Abitur im fünften Jahr nach dem Studium betrachtet. Der Berufsstatus einer Person ist von 
Bedeutung, da ihm ein besonderer gesellschaftlicher Stellenwert zukommt. Er verortet seinen Inhaber in der gesellschaftlichen Hierarchie und eröffnet Lebenschancen: Die Gesellschaft honoriert den erreichten Status mit entsprechender Entlohnung (Erikson 1992).

Der Artikel ist wie folgt gegliedert: In Abschnitt 2 werden die Berufsplatzierungen vorgestellt, die Hochschulabsolventen erwarten können, sowie die Determinanten mit Fokus auf die soziale Herkunft vorgestellt. Das Motiv der Statusreproduktion wird für die Erklärung von Unterschieden in der Statusplatzierung der Absolventen ohne und mit Abitur vorgestellt und daraus empirisch überprüfbare Hypothesen abgeleitet. Abschnitt 3 beschreibt die verwendeten Daten, ihre Operationalisierung sowie die Analysestrategie. Die Befunde werden in Abschnitt 4 vorgetragen. Im Fazit werden die Erkenntnisse des Artikels zusammengefasst und diskutiert.

\section{Soziale Herkunft und Statusplatzierungen}

Der Einfluss der sozialen Herkunft auf die Statusplatzierungen kann durch primäre und sekundäre Herkunftseffekte erklärt werden (Boudon 1974). Das Leistungsniveau der Kindergeneration wird im Rahmen von primären Herkunftseffekten von höheren kognitiven und monetären Ressourcen der statushöheren Eltern beeinflusst und selbst leistungsschwache Kinder können so erfolgreich an höherer Bildung partizipieren. Die Bildungsaspirationen von Eltern und ihren Kindern wirken als sekundäre Herkunftseffekte. Über die strukturelle Verankerung der Eltern in der gesellschaftlichen Sozialstruktur unterscheiden sich diese Aspirationen systematisch voneinander. Die Position der Eltern in der Gesellschaft reflektiert die Lebenschancen, die die Kindergeneration seit Geburt an kennengelernt hat und die über den eigenen Bildungsweg reproduziert werden soll. Zwischen den Bildungserträgen, die mit einem Hochschulabschluss zu erwarten sind, und dem Status der Eltern besteht eine soziale Distanz, deren Länge mit dem Ausmaß zunimmt, je statusniedriger die Elternhäuser sind. ${ }^{1}$ Gleichzeitig sind die Aspirationen, die für die Überbrückung der sozialen Distanz notwendig sind, über alle Schichten normalverteilt und zur Wahrung der im Elternhaus erfahrenen Lebenschancen

\footnotetext{
${ }^{1}$ Für den Gedanken der sozialen Distanz greift Boudon (1974) auf Keller/ Zavalloni (1964) zurück.
} 
Soziale Herkunft, Statusreproduktion und Studium ohne Abitur

streben Eltern für ihre Kinder die Reproduktion des eigenen Status an. Dies führt zu den herkunftsbezogenen Unterschieden in den gewählten Bildungswegen.

Breen und Goldthorpe (1997) formalisieren den Gedanken der sekundären Herkunftseffekte für die Wahl zwischen dem Verbleib im Bildungssystem und dem Eintritt in den Arbeitsmarkt. Für die Autoren ist zentral, dass Eltern für ihre Kinder den Bildungsweg wählen, der ihnen die intergenerationale Statusreproduktion sichert. Sobald die Schülerinnen und Schüler den hierfür notwendigen Bildungsabschluss erreicht haben, verlassen sie das Bildungssystem und treten in den Arbeitsmarkt ein. Grund dafür ist, dass ein Verbleib im Bildungssystem mit dem Risiko des Scheiterns und einem sozialen Abstieg verbunden wäre. Die Vermeidung des sozialen Abstiegs führt bei Schülerinnen und Schülern aus statusniedrigeren Elternhäusern dazu, dass sie früher das Bildungssystem verlassen und von der formalen Hochschulzugangsberechtigung abgelenkt werden. Dagegen müssen Schülerinnen und Schüler aus statushohen Elternhäusern länger in ihre Bildung investieren, um dann mit dem Hochschulabschluss den elterlichen Status auf dem Arbeitsmarkt reproduzieren zu können. Für Akademiker mit Abitur führt dies dazu, dass ihre soziale Herkunft auf ihre späteren Statusplatzierungen wirkt und dass ein statushohes Elternhaus auch häufiger zu einer statushohen Platzierung führt, im Unterschied zu einem statusmittleren Elternhaus oder einem statusniedrigen Elternhaus.

Wendet man die Überlegungen von Boudon (1974) sowie von Breen und Goldthorpe (1997) auf die Erklärung des Zusammenhangs zwischen sozialer Herkunft und eigener Statusreproduktion an, ist zu klären, warum Akademiker ohne Abitur aus statusmittleren und statusniedrigeren Elternhäusern trotz bereits erfolgreich abgeschlossener Statusreproduktion ein Studium ohne Abitur aufnehmen. Zwei Faktoren spielen dabei eine Rolle: die soziale Distanz und die normalverteilte Motivation, also die gleiche Motivation in jeder Schicht, den Status der Eltern zu reproduzieren.

Die soziale Distanz hin zu den beruflichen Statuspositionen eines Studienabschlusses ist für Akademiker ohne Abitur aus statusniedrigen und statusmittleren Elternhäusern häufig zu groß, angesichts einer normalverteilten Motivation. Ihre Wahrscheinlichkeit ein Studium aufzunehmen sollte steigen, wenn sie sich nach der Berufsausbildung von dem Berufsstatus im Elternhaus durch berufliche Aufwärtsmobilität lösen und ihren eigenen Berufsstatus als Schwellenwert für die Statusreproduktion als 
Akademiker ohne Abitur etablieren. Sowohl für Akademiker ohne Abitur aus statusniedrigeren als auch aus statusmittleren Elternhäusern führt die berufliche Aufwärtsmobilität dazu, bessere Berufspositionen einzunehmen, sodass die soziale Herkunft keinen Einfluss mehr auf die Platzierung in der Berufshierarchie nach dem Studium hat. Dies wird bei Akademiker ohne Abitur aus statusniedrigeren Elternhäusern durch eine überdurchschnittliche Motivation gefördert, die sie benötigen, um die für sie größere soziale Distanz im Vergleich zu den Akademikern aus allen anderen Schichten zu überbrücken. Es kann erwartet werden, dass sie als Akademiker ohne Abitur nicht nur den eigenen Status vor dem Studium reproduzieren, sondern nach höheren Positionen in der Berufshierarchie streben.

Dagegen reproduzierten Akademiker ohne Abitur aus statushohen Elternhäusern den Berufsstatus im Elternhaus durch die Berufsausbildung nicht. Sie wurden trotz günstiger primärer Herkunftseffekte von einer weiterführenden Bildungskarriere und einem Hochschulstudium abgelenkt, da sie nicht das Leistungsniveau für eine allgemeine Hochschulzugangsberechtigung erreichen. Basierend auf der Annahme, dass sie weiterhin durch das auf dem ersten Bildungsweg vorrangige Bestreben nach Statusreproduktion motiviert sind, bietet ihnen das Studium ohne Abitur die Möglichkeit, den elterlichen Status als Akademiker anzustreben. Ihre soziale Herkunft sollte weiterhin einen starken Einfluss auf ihre Statusplatzierung haben.

\section{Daten und Operationalisierung}

\subsection{Daten}

Die folgenden Analysen basieren auf Daten der Erwachsenenkohorte SC6 7.0.0 des Nationalen Bildungspanels, NEPS (Blossfeld et al. 2011). Das NEPS ist ein Datensatz, der Informationen über bestimmte Bildungsereignisse abfragt. Der Survey besteht aus sechs Startkohorten, die alle Bildungsetappen eines Menschen nachzeichnen. Die Erwachsenenbefragung wurde ursprünglich 2007/2008 vom Institut für Arbeitsmarkt- und Berufsforschung (IAB) als Studie „Arbeiten und Lernen im Wandel (ALWA)“ durchgeführt. Für die Befragten der Erwachsenenkohorte liegen (retrospektive) Erwerbsinformationen von 1963 bis 2015 vor. 
Soziale Herkunft, Statusreproduktion und Studium ohne Abitur

Betrachtet wird die Statusplatzierung im fünften Jahr nach dem Fachhochschul- oder Universitätsabschluss. Alle Absolventen haben entweder vor 1991 ein Studium in Westdeutschland beziehungsweise seit 1991 in Gesamtdeutschland aufgenommen. Selbstständige sind aus der Analyse ausgeschlossen. Als Akademiker ohne Abitur gelten Befragte, die ohne Abitur aber mit beruflicher Qualifikation und über eine Eignungsprüfung ein Studium an einer Fachhochschule oder einer Universität aufgenommen haben. Dagegen haben Akademiker mit Abitur aber ohne berufliche Vorausbildung, ein Studium aufgenommen, d. h. Mehrfachqualifizierer sind aus der Stichprobe ausgeschlossen. Das Analysesample umfasst 146 Akademiker ohne Abitur und 628 Akademiker mit Abitur.

\subsection{Variablen}

Der berufliche Status wird anhand des International Socio-Economic Index of Occupational Status (ISEI) von Ganzeboom und Treiman (2010) operationalisiert. Der ISEI-08 rangiert von 11,74 Statuspunkten für land- und tierwirtschaftliche Arbeitskräfte bis hin zu 88,96 für Ärzte oder Richter.

Für den Unterschied zwischen den Akademikern ohne und mit Abitur wird ein dichotomer Indikator mit 1 = Akademiker ohne Abitur und $0=$ Akademiker mit Abitur auf Grundlage der Frage Auf welchem Weg haben Sie die Studienberechtigung erworben? Und der Antwort als beruflich Qualifizierter generiert.

Der höchste berufliche Status im Elternhaus (HISEI) wird ebenfalls über den ISEI-08 operationalisiert. Für den HISEI wird der ISEI in einen niedrigen, mittleren und hohen beruflichen Status kategorisiert, um die idealtypische Schichtung der Gesellschaft, wie sie in der Argumentation aufgenommen wurde, abzubilden. Ein niedriger beruflicher Status entspricht den unteren 25 Prozent der elterlichen ISEI-Verteilung im vorliegenden Sample. Das mittlere Prestige entspricht allen Werten über dem 25 Prozent-Schnittpunkt bis hin zu unter 75 Prozent der Verteilung. Alle Werte im oberen Quartil der Verteilung werden einem hohen beruflichen Berufsstatus zugeordnet.

Zudem wird für die Bildungsindikatoren Universität versus Fachhochschule, die Besetzung einer Leitungsposition versus keiner Leitungsposition, Vertragsbefristung versus Vertragsentfristung, der Arbeitsort (Ost- versus Westdeutschland) sowie Alter, Geschlecht und Jahr kontrolliert. Eine Übersicht 
der Rekodierungen für die Analyse mit zugehörigen Mittelwerten und Standardabweichungen sowie den Häufigkeitsverteilungen der verwendeten Variablen findet sich im Anhang in Tabelle A1.

\section{Befunde}

\subsection{Deskriptive Ergebnisse}

Der folgende Abschnitt stellt die Akademiker ohne und mit Abitur vor, die fünf Jahre nach dem Studienabschluss in der Analysestichprobe vorhanden sind. Die Mehrheit der Akademiker ohne Abitur ist männlich (63,0\%). Sie sind im Durchschnitt 37,4 Jahre alt, allerdings variiert ihr Alter stark um eine Standardabweichung von 9,28. Akademiker ohne Abitur haben vorwiegend an der Fachhochschule $(71,2 \%)$ und nicht an einer Universität studiert. Zum betrachteten Zeitpunkt sind sie größtenteils vertraglich entfristet $(88,4 \%)$ und sind in leitender Position tätig $(67,1 \%)$. Die Akademiker mit Abitur unterscheiden sich zum Teil von den Akademikern ohne Abitur. Zwar sind auch sie überwiegend männlich (55,3\%). Sie sind aber im Durchschnitt gut vier Jahre jünger. Akademiker mit Abitur haben zudem häufiger an einer Universität studiert (83,3 \%). Beruflich sind sie ebenfalls häufiger als Akademiker ohne Abitur vertraglich entfristet $(77,4 \%)$ und arbeiten häufiger als diese in leitender Position $(70,2 \%)$.

Akademiker ohne Abitur kommen häufiger aus statusniedrigen $(34,3 \%)$ und statusmittleren $(46,6 \%)$ und seltener aus statushohen (19,2\%) Elternhäusern, im Vergleich dazu kommen Akademiker mit Abitur vorwiegend aus statushohen $(29,6 \%)$ und statusmittleren $(48,6 \%)$ und seltener aus statusniedrigeren (21,8\%) Elternhäusern. Abbildung 1 stellt die Ergebnisse des Mittelwertvergleichs im fünften Jahr nach Studienabschluss für die Gesamtstichprobe, sowie getrennt nach der Statuszugehörigkeit des Elternhauses (statusniedrig, statusmittel und statushoch) dar. Wie die Mittelwertvergleiche zeigen, sind Akademiker ohne Abitur sowohl in der Gesamtstichprobe als auch innerhalb der einzelnen Schichten signifikant niedriger in der Berufshierarchie platziert als die Akademiker mit Abitur. 
Abbildung 1: Mittelwerte der Statusplatzierungen, Akademiker ohne und mit Abitur (|t|-Werte über den Pfeilen und ISEI-Werte in den Balken)

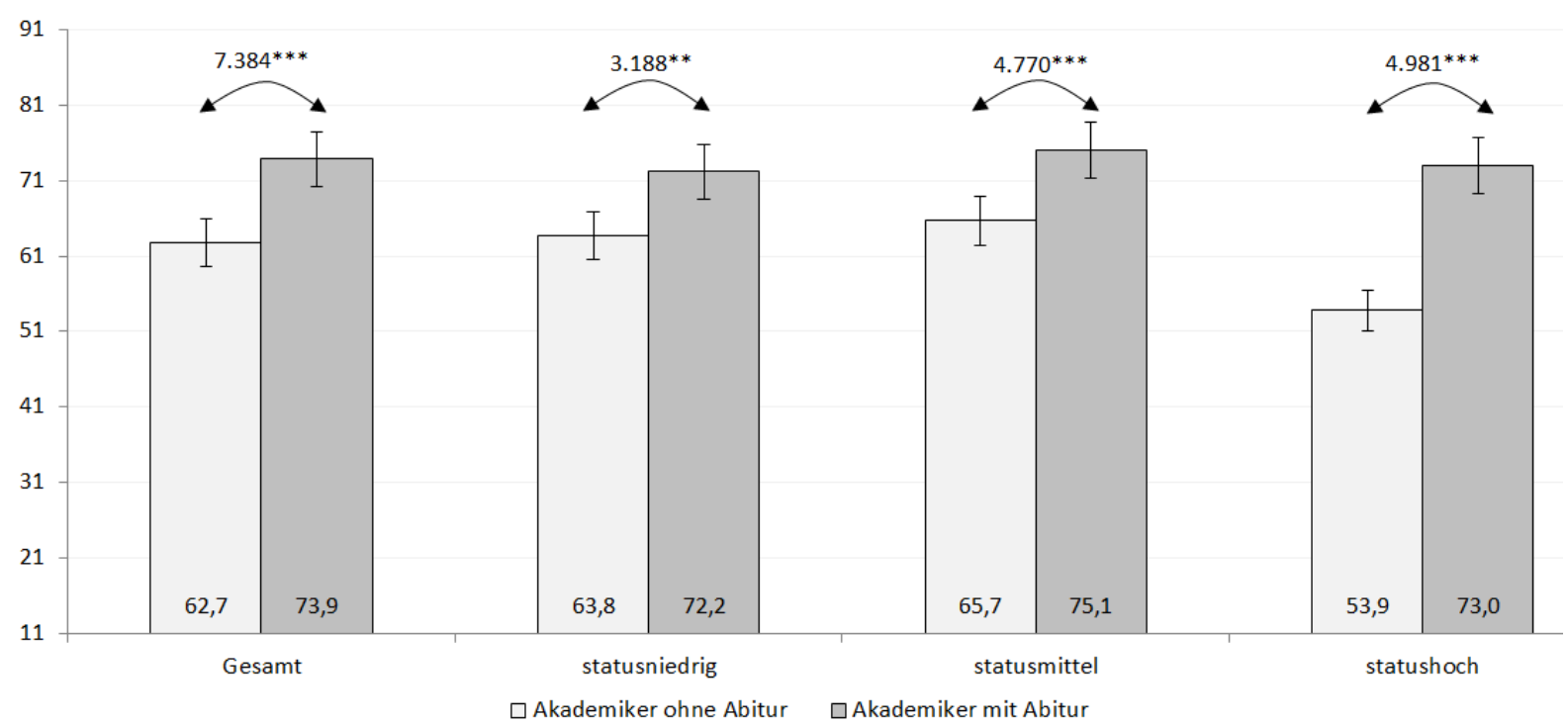

Angegeben sind Mittelwerte an der Basis der Balken sowie die $|\mathrm{t}|$-Werte über den Pfeilen mit $\mathrm{p}<0,05=*$, $\mathrm{p}<0.01=* *$ und $\mathrm{p}<0.001=* * * . \mathrm{N}=733$.

Quelle: LIfBi, NEPS, Startkohorte 6, 2007-2015, doi:10.5157/NEPS:SC6:7.0.0

Die deskriptiven Analysen geben Hinweise darauf, dass sich die Akademiker ohne und mit Abitur hinsichtlich der Rolle ihrer sozialen Herkunft voneinander unterscheiden. Besonders auffällig ist die Statusdifferenz zwischen den Akademikern ohne und mit Abitur aus statushohen Elternhäusern. Fünf Jahre nach dem Studium zeigt sich, dass insbesondere Akademiker ohne Abitur aus statushohen Elternhäusern statusniedriger in der Berufshierarchie platziert sind als Akademiker mit Abitur derselben Herkunftsgruppe. Dennoch bleibt offen, ob sich der Effekt der sozialen Herkunft auf die Statusplatzierungen verliert, wenn für andere Faktoren kontrolliert wird. Dieser Frage wird in den multivariaten Analysen nachgegangen.

\subsection{Multivariate Analysen}

Inwiefern der Einfluss der sozialen Herkunft auf die Statusplatzierungen der Akademiker ohne Abitur wirkt, zeigen die schrittweise erweiterten linearen Regressionsmodelle. Angegeben sind bKoeffizienten mit dem Standardfehler in Klammern. Die b-Koeffizienten können als die Anzahl von ISEI-Statuspunkten gelesen werden, die die Zugehörigkeit zu der jeweiligen Variable den Berufsstatus 
der Akademiker ohne und mit Abitur verändert. Tabelle 1 zeigt die Ergebnisse der Regressionsmodelle.

In Modell M1 ist der Zusammenhang zwischen der sozialen Herkunft und der beruflichen Statusplatzierung der Akademiker mit Abitur dargestellt. Mit einem R2 von 0.009 erklärt die soziale Herkunft knapp ein Prozent der Statusplatzierungen der Akademiker mit Abitur. Gemessen an Personen aus statusniedrigen Elternhäusern sind nur Personen aus statusmittleren Elternhäusern statushöher in der Berufshierarchie platziert. Modell M2 kontrolliert den Zusammenhang der sozialen Herkunft aus Modell M1 um ausgewählte Personenmerkmale (Alter, Geschlecht) sowie um die Art der Hochschule, berufliche Rahmenbedingungen (Arbeitsort in Ostdeutschland, Tätigkeit in einer Leitungsposition und Befristung des Arbeitsvertrags) und das Kalenderjahr, in dem das fünfte Jahr nach dem Studium stattgefunden hat. Das Modell M2 erklärt 11,7 \% der Statusplatzierungen der Akademiker mit Abitur. Der Einfluss der mittleren sozialen Herkunft wird unter Kontrolle dieser Faktoren für die Akademiker mit Abitur aus statusmittlerem Elternhaus stärker, der Trend im Einfluss der sozialen Herkunft bleibt jedoch gleich. Universitätsabsolventen mit Abitur sind höher als Fachhochschulabsolventen in der Berufshierarchie platziert, während die Entfristung des Beschäftigungsverhältnisses und der zeitlich spätere Abschluss eines Studiums mit schlechteren Statusplatzierungen einhergehen. 
Soziale Herkunft, Statusreproduktion und Studium ohne Abitur

Tabelle 1: Beruflicher Status fünf Jahre nach Studienabschluss (lineare Regression)

\begin{tabular}{|c|c|c|c|c|c|}
\hline & \multicolumn{2}{|c|}{ Akademiker mit Abitur } & \multicolumn{3}{|c|}{ Akademiker ohne Abitur } \\
\hline & Modell (M1) & Modell (M2) & Modell (M3) & \multicolumn{2}{|c|}{ Modell (M4) } \\
\hline Mittlerer Status & $2.909 *(1.35)$ & $3.320 * *(1.29)$ & $1.945 \quad(3.04)$ & -0.666 & $(2.97)$ \\
\hline Hoher Status & $0.741(1.48)$ & $1.308 \quad(1.43)$ & $-8.460 *(3.86)$ & $-12.251 * * *$ & (3.77) \\
\hline Alter & & $-0,172 \quad(0.97)$ & & -0.060 & $(0.19)$ \\
\hline $\begin{array}{l}\text { Geschlecht } \\
\text { (Ref.: weiblich) }\end{array}$ & & $-0.984 \quad(1.04)$ & & 2.005 & (2.81) \\
\hline $\begin{array}{l}\text { Universität } \\
\text { (Ref. Fachhochschule) }\end{array}$ & & $9.416 * * *(1.40)$ & & 3.424 & $(2.92)$ \\
\hline $\begin{array}{l}\text { Arbeitstätigkeit in Ost- } \\
\text { DE (Ref.:West-DE) }\end{array}$ & & $-2.391 \quad(2.10)$ & & 4.948 & (3.68) \\
\hline $\begin{array}{l}\text { Leitungsposition (Ref. } \\
\text { Keine) }\end{array}$ & & $0.061 \quad(1.13)$ & & -0.905 & $(2.84)$ \\
\hline $\begin{array}{l}\text { Befristung (Ref. Keine } \\
\text { Befristung) }\end{array}$ & & $-3.416 * *(1.21)$ & & $11.533^{* *}$ & (4.29) \\
\hline Zeit & & $-0.112 *(1.20)$ & & $-0.385^{* *}$ & $(0.14)$ \\
\hline Konstante & $72.297 * * *(1.12)$ & $68.322 * * *(2.02)$ & $63.780 * * *(2.31)$ & $54.638 * * *$ & (4.99) \\
\hline $\mathrm{R}^{2}$ & 0.009 & 0.117 & 0.054 & 0.20 & \\
\hline $\mathrm{N}$ & & 8 & & 46 & \\
\hline
\end{tabular}

Die Modelle M3 und M4 verdeutlichen für die Akademiker ohne Abitur, dass hier die soziale Herkunft anders auf die eigenen Statusplatzierungen wirkt als dies bei Absolventen mit Abitur der Fall ist. Zum einen erklärt die soziale Herkunft gut 5 Prozent der Statusplatzierungen (M3). Zum anderen ist die soziale Herkunft insofern von Bedeutung, als Akademiker ohne Abitur aus statushohen Elternhäusern gegenüber solchen aus statusniedriger Herkunft eine niedrigere Platzierung in der Berufshierarchie erreichen. Dieser negative Platzierungsunterschied ist - wie in den durchgeführten Mittelwertunterschieden - mit fast 8,5 ISEI-Punkten hoch. Unter Kontrolle der bereits erwähnten Merkmale, verbessert sich die Erklärung der Statusplatzierungen der Akademiker ohne Abitur auf 20,1 Prozent (M4). Der berufliche Status der Akademiker ohne Abitur aus statushohem Elternhaus verschlechtert sich um 12,3 Statuspunkte im Vergleich zu solchen aus statusniedrigen Elternhäusern. Im Gegensatz zu den Akademikern mit Abitur wirkt ein unbefristeter Arbeitsvertrag für die Akademiker ohne Abitur positiv auf die Statusplatzierungen. 


\section{Diskussion}

In dem vorliegenden Aufsatz wurde der Frage nachgegangen, ob die soziale Herkunft auch nach einem erfolgreich abgeschlossenen Studium noch einen Einfluss auf die Bildungserträge von Akademikern hat. Dieser Frage wurde anhand der Statusplatzierungen von Akademikern ohne und mit Abitur in der Berufshierarchie nachgegangen. Es wurde nach Boudon (1974) sowie Breen und Goldthorpe (1997) angenommen, dass der Einfluss der sozialen Herkunft auf die Statusplatzierungen der Akademiker ohne Abitur aus statusmittleren und statusniedrigeren Schichten nicht vorhanden ist. Als Grund hierfür wurde angenommen, dass sich diese Akademiker ohne Abitur vor dem Studium von ihrem Elternhaus lösen und ihre soziale Distanz zu den akademischen Bildungserträgen verringern. Es ist daher ihre Motivation, mit dem Studium, ihren eigenen Status zu reproduzieren. Ihre soziale Herkunft, so wurde weiterhin angenommen, hat daher keinen weiteren Einfluss auf die Statusplatzierungen nach dem Studium. Dagegen können Akademiker ohne Abitur aus statushohen Elternhäusern vor dem Studium ohne Abitur den elterlichen Status, trotz günstiger primärer Herkunftseffekte, nicht reproduzieren. Daher - so wurde weiter angenommen - hat die soziale Herkunft in ihrem Fall weiterhin einen Einfluss auf ihre Statusplatzierungen. Für Akademiker mit Abitur wurde angenommen, dass das Motiv der Statusreproduktion für die spätere Statusplatzierung zentral ist und es daher zu einer hierarchischen Schichtung der Platzierung nach dem Berufsstatus im Elternhaus kommt.

Analysen mit der Erwachsenenkohorte SC6 7.0.0 des NEPS konnten zeigen, dass die Chancengleichheit der Öffnung der Hochschulen für Akademiker ohne Abitur nur zu einer partiellen Lösung des Zusammenhangs von sozialer Herkunft und Bildungserträgen führt: Für Akademiker ohne Abitur aus statushohen Elternhäusern wirkt ihre soziale Herkunft weiterhin auf die eigenen Statusplatzierungen nach dem Studium, allerdings stehen diese - nicht wie bei den Akademikern mit Abitur - in einem negativen Zusammenhang mit den Statusplatzierungen. Dagegen sind es die vormals von ihrer sozialen Herkunft benachteiligten Akademiker ohne Abitur aus statusniedrigen und statusmittleren Elternhäusern, die vom Studium ohne Abitur profitieren. Ihre soziale Herkunft wirkt nicht mehr auf ihre Statusplatzierungen. Dennoch kommt es nicht zu einer Ergebnisgleichheit der Statusplatzierungen mit den Akademikern mit Abitur. Vielmehr sind die Akademiker ohne Abitur aus statusniedrigem 
Soziale Herkunft, Statusreproduktion und Studium ohne Abitur

und statusmittlerem Elternhaus im fünften Jahr nach dem Studienabschluss vergleichsweise niedriger in der Berufshierarchie platziert. Soziale Disparitäten, die früher im allgemeinen Bildungssystem wirkten und sich in den letzten Jahren sukzessive in das tertiäre Bildungssystem verlagerten, haben sich mit der Durchsetzung der nachträglichen Chancengleichheit im Zugang zu Hochschulbildung in den Arbeitsmarkt verschoben. 


\section{Literaturverzeichnis}

Allmendinger, J. (1989): Educational systems and labor market outcomes, in: European Sociological Review 5 (3), 231-250.

Baumert, J./Schümer, G. (2001): Familiäre Lebensverhältnisse, Bildungsbeteiligung und Kompetenzerwerb, in: Deutsches PISA-Konsortium (Hg.), PISA 2000. Basiskompetenzen von Schülerinnen und Schülern im internationalen Vergleich, Opladen, 323-407.

Blossfeld, H.-P./Roßbach, H.-G./Maurice, J. von (Hg.) (2011): Education as a Lifelong Process. The German National Educational Panel Study (NEPS), in: Zeitschrift für Erziehungswissenschaft, 14 (Sonderheft 2), Wiesbaden, VS.

Boudon, R. (1974): Education, opportunity, and social inequality, Changing prospects in Western society, New York and London and Sydney and Toronto, John Wiley.

Brändle, T. (2016): How availability of capital affects the timing of enrollment: the route to university of traditional and non-traditional students, In: Studies in Higher Education, 1-21.

Brändle, T./Lengfeld, H. (2015): Erzielen Studierende ohne Abitur geringeren Studienerfolg? Befunde einer quantitativen Fallstudie, In: Zeitschrift für Soziologie 44 (6), 447-467.

Brändle, T./Lengfeld, H. (2017): Drifting apart or converging? Grades among non-traditional and traditional students over the course of their studies: a case study from Germany, In: Higher Education (73 (2)), 227-244.

Breen, R./Luijkx, R./Müller, W./Pollak, R. (2009): Nonpersistent Inequality in Educational Attainment: Evidence from Eight European Countries, In: American Journal of Sociology 114, 1475-1521.

- (2010): Long-term trends in educational inequality in Europe: Class inequalities and gender differences, In: European Sociological Review 26 (1), 31-48.

- (2012): Bildungsdisparitäten nach sozialer Herkunft und Geschlecht im Wandel: Deutschland im internationalen Vergleich, In: Becker, R./Solga/H. (Hrsg.): Soziologische Bildungsforschung, Bd. 52. Wiesbaden: Springer VS Verlag (Sonderheft der Kölner Zeitschrift für Soziologie und Sozialpsychologie, 52), 346-373.

Breen, R./Goldthorpe, J.H. (1997): Explaining educational differentials: towards a formal rational action theory, in: Rationality and Society 9 (3), 275-305.

Dahm, G./Kerst, C. (2016): Erfolgreich studieren ohne Abitur? Ein mehrdimensionaler Vergleich des Studienerfolgs von nicht-traditionellen und traditionellen Studierenden, In: Wolter, A./ Banscherus, U./ Kamm, C.: Zielgruppen Lebenslangen Lernens an Hochschulen, 225-265.

Destatis (2016): Bestandene Prüfungen nach Art der HZB (1997 bis 2015), Hg. v. Statistisches Bundesamt Deutschland, Wiesbaden.

Diller, F./Festner, D./Freiling, T./Huber, S. (2011): Qualifikationsreserven durch Quereinstieg nutzen: Studium ohne Abitur, Berufsabschluss ohne Ausbildung, Bielefeld: Bertelsmann Verlag.

Ehmke, T. /Baumert, J. (2007): Soziale Herkunft und Kompetenzerwerb: Vergleiche zwischen PISA 2000, 2003 und 2006, In: Prenzel et al. (2007), 309-335.

Erikson, R./Goldthorpe, J. H. (1992): The Constant Flux. A Study of Class Mobility in Industrial Societies, Oxford: Clarendon Press.

Ganzeboom, H.B.G./Treiman, D.J. (2010): Occupational status measures for the new international standard classification of occupations ISCO-08, With a discussion of the new classification, VU University Department of Social Research Methodology, Amsterdam.

Hartmann, M./Kopp, J. (2001): Elitenselektion durch Bildung oder durch Herkunft? In: Kölner Zeitschrift für Soziologie und Sozialpsychologie, 53, 436-466. 
Keller, S./Zavalloni, M. (1964): Ambition and social class: A respecification, In: Social Forces 43 (1), $58-70$.

KMK (2009): Hochschulzugang für beruflich Qualifizierte ohne schulische Hochschulzugangsberechtigung, KMK.

Lörz, M. (2013): Differenzierung des Bildungssystems und soziale Ungleichheit: Haben sich mit dem Ausbau der beruflichen Bildungswege die Ungleichheitsmechanismen verändert? In: Zeitschrift für Soziologie 42 (2).

Lörz, M./Schindler, S. (2011): Bildungsexpansion und soziale Ungleichheit: Zunahme, Abnahme oder Persistenz ungleicher Chancenverhältnisse - eine Frage der Perspektive? In: Zeitschrift für Soziologie 40 (6), 458-477.

Maaz, K./ Baumert, J./ Cortina, K.S. (2008): Soziale und regionale Ungleichheiten im deutschen Bildungssystem, In: Cortina, K.S./ Baumert, J./ Leschinsky, A./ Mayer, K.U./ Trommer, L. (Hg.), Das Bildungswesen in der Bundesrepublik Deutschland, Hamburg: Rowohlt Verlag GmbH, 205-243.

Maaz, K./Baumert, J./Trautwein, U. (2009): Genese sozialer Ungleichheit im institutionellen Kontext der Schule: Wo entsteht und vergrößert sich soziale Ungleichheit? In: Zeitschrift für Erziehungswissenschaft. Sonderheft 12: Bildungsentscheidungen, 11-46.

Maaz, K. /Dumont, H. (2018): Ungleichheiten des Bildungserwerbs nach sozialer Herkunft, Migrationshintergrund und Geschlecht, In: Köller, O./Hasselhorn, M./Hesse, F./Maaz, K./Schrader, J./Solga, H./Spieß, C.K./Zimmer, K. (2018): Das Bildungswesen in Deutschland, Bestand und Potenziale, Bad Heilbrunn: Klinkhardt.

Manzoni, A./Harkonen, J./ Mayer, K.U. (2014): Moving on? A Growth-Curve Analysis of Occupational Attainment and Career Progression Patterns in West Germany, In: Social Forces 92, 1285 - 1312.

Müller, W. (2001): Zum Verhältnis von Bildung und Beruf in Deutschland, Entkopplung oder zunehmende Strukturierung? In: Berger, P.A./Konietzka, D. (Hrsg.), Die Erwerbsgesellschaft, Wiesbaden: Springer, 29-63.

Müller, W./Gangl, M. (Hrsg.) (2003): Transitions from education to work in Europe: The integration of youth into EU labour market, Oxford: Oxford University Press.

Müller, W./Jacob, M. (2008): Qualifications and the returns to training across the life course.

Müller, W./Pollak, R. (2010): Weshalb gibt es so wenige Arbeiterkinder in Deutschlands Universitäten? In: Becker, R./ Lauterbach, W. (Hg.): Bildung als Privileg? Erklärungen und Befunde zu den Ursachen von Bildungsungleichheit, Wiesbaden: VS Verlag für Sozialwissenschaften, 305-344.

Müller, W./Pollak, R./Reimer, D./Schindler, S. (2011): Hochschulbildung und soziale Ungleichheit, in: Becker, R. (Hg.): Lehrbuch der Bildungssoziologie, Wiesbaden: VS Verlag für Sozialwissenschaften, 289-327.

Nickel, S./Duang, S. (2012): Studieren ohne Abitur, Monitoring der Entwicklung in Bund, Ländern und Hochschulen, Centrum für Hochschulentwicklung, Gütersloh.

Rzepka, S. (2016): Labor market returns to college education with vocational qualifications, Bochum (Ruhr Economic Papers).

Schindler, S./Reimer, D. (2010): Primäre und sekundäre Effekte der sozialen Herkunft beim Übergang in die Hochschule, in: Kölner Zeitschrift für Soziologie und Sozialpsychologie 62 (4), 623-653.

Schindler, S./Lörz, M. (2012): Mechanisms of social inequality development: Primary and secondary effects in the transition to tertiary education between 1976 and 2005, in: European Sociological Review 28 (5), 647-660. 
Teichler, U./Wolter, A. (2004): Zugangswege und Studienangebote für nicht-traditionelle Studierende, in: die Hochschule, 2/2004, 64-80.

Ulbricht, L. (2012): Öffnen die Länder ihre Hochschulen? Annahmen über den Dritten Bildungsweg auf dem Prüfstand, in: DieHochschule, Jg. 13, Heft 2, 64-80.

Watermann, R./Daniel, A./Maaz, K. (2014): Primäre und sekundäre Disparitäten des Hochschulzugangs: Erklärungsmodelle, Datengrundlagen und Entwicklungen, in: Kai Maaz, Marko Neumann und Jürgen Baumert (Hg.): Herkunft und Bildungserfolg von der frühen Kindheit bis ins Erwachsenenalter. Forschungsstand und Interventionsmöglickeiten aus interdisziplinärer Perspektive. Wiesbaden: Springer VS, 233-261.

Wolter, A./Banscherus, U./Kamm, C./Otto, A./Spexard, A. (2014): Durchlässigkeit zwischen beruflicher und akademischer Bildung, in: Beiträge zur Hochschulforschung, 36. Jahrgang, 4/2014.

Wolter, A./Dahm, G./Kamm, C./Otto, A. (2015): Nicht-traditionelle Studierende in Deutschland: Werdegänge und Studienmotivation, in: Elsholz, U. (Hg.): Beruflich Qualifizierte im Studium: Analysen und Konzepte zum Dritten Bildungsweg. Bielefeld: W. Bertelsmann Verlag, 11-34.

Diese Arbeit nutzt Daten des Nationalen Bildungspanels (NEPS): Startkohorte Erwachsene, doi:10.5157/NEPS:SC6:7.0.0. Die Daten des NEPS wurden von 2008 bis 2013 als Teil des Rahmenprogramms zur Förderung der empirischen Bildungsforschung erhoben, welches vom Bundesministerium für Bildung und Forschung (BMBF) finanziert wurde. Seit 2014 wird NEPS vom Leibniz-Institut für Bildungsverläufe e.V. (LIfBi) an der Otto-Friedrich-Universität Bamberg in Kooperation mit einem deutschlandweiten Netzwerk weitergeführt. 
Soziale Herkunft, Statusreproduktion und Studium ohne Abitur

\section{Anhang}

Tabelle A1 Variablenbeschreibung der Analysestichprobe

\begin{tabular}{|c|c|c|c|c|}
\hline Bezeichnung & Originalskalierung & Rekodierung & a.M. (SD) & $\begin{array}{l}\text { Rel. Häu- } \\
\text { figk. }\end{array}$ \\
\hline \multicolumn{5}{|l|}{ Abhängige Variable } \\
\hline $\begin{array}{l}\text { International Socio- } \\
\text { Economic Index of Oc- } \\
\text { cupational Status (ISEI- } \\
\text { 2008) }\end{array}$ & $11,74-88,96$ & metrisch & $71,8(14,5)$ & \\
\hline \multicolumn{5}{|l|}{ Unabhängige Variablen } \\
\hline $\begin{array}{ll}\text { Höchster } & \text { beruflicher } \\
\text { Status im } & \text { Elternhaus }\end{array}$ & $11,74-88,96$ & $\begin{array}{l}0-\text { niedriger Status ( }<=25 \text { Pro- } \\
\text { zent) }\end{array}$ & & $24,2 \%$ \\
\hline \multirow[t]{2}{*}{ (ISEI-2008) } & & $\begin{array}{l}1-\text { mittlerer Status ( }>25 \text { Prozent } \\
\text { und }<75 \text { Prozent) }\end{array}$ & & $48,2 \%$ \\
\hline & & $2-$ hoher Status (>=75 Prozent) & & $27,7 \%$ \\
\hline Geschlecht & & $0=$ weiblich; 1 = männlich & & $1=73,0 \%$ \\
\hline Alter & & $\begin{array}{l}\text { Offen, für die multivariate Analyse } \\
\text { zentriert }\end{array}$ & $33,9(6,8)$ & \\
\hline $\begin{array}{l}\text { Universität } \\
\text { (Ref. Fachhochschule) }\end{array}$ & & $\begin{array}{l}0=\text { Fachhochschule; } 1=\text { Universi- } \\
\text { tät }\end{array}$ & & $1=43,3 \%$ \\
\hline $\begin{array}{l}\text { Arbeitstätigkeit in Ost- } \\
\text { deutschland (Ref. West- } \\
\text { deutschland) }\end{array}$ & & $\begin{array}{l}0=\text { Ostdeutschland, } 1=\text { West- } \\
\text { deutschland }\end{array}$ & & $1=8,9 \%$ \\
\hline $\begin{array}{l}\text { Leitungsposition (Ref.: } \\
\text { keine Leitungsposition) }\end{array}$ & & $\begin{array}{l}0=\text { keine Leitungsposition; } 1= \\
\text { Leitungsposition }\end{array}$ & & $1=69,6 \%$ \\
\hline $\begin{array}{l}\text { Befristung (Ref. keine } \\
\text { Befristung) }\end{array}$ & & $\begin{array}{l}0=\text { unbefristeter Arbeitsvertrag; } 1 \\
=\text { befristeter Arbeitsvertrag }\end{array}$ & & $1=79,5 \%$ \\
\hline Kalenderjahr & & $1968-2015$ & $1994(12.7)$ & \\
\hline
\end{tabular}

Anmerkungen: $\mathrm{N}=774$ Akademiker ohne und mit Abitur. Ref. steht für Referenzgruppe.

Quelle: LIfBi, NEPS, Startkohorte 6, 2007-2015, doi:10.5157/NEPS:SC6:7.0.0 